\title{
Comparative Analysis of Traditional Methods with Game of Life in Cellular Automata for Edge Detection
}

\author{
Sukriti Mehrotra \\ Dept of Computer Sc. and Engg. \\ Sikkim Manipal Institute of Technology \\ Majitar

\section{Shilpi Shikha} \\ Dept of Computer Sc. and Engg. \\ Sikkim Manipal Institute of Technology \\ Majitar
}

\author{
Sohini Das \\ Dept of Computer Sc. and Engg. \\ Sikkim Manipal Institute of Technology \\ Majitar \\ Chinmoy Kar \\ Dept of Computer Sc. and Engg. \\ Sikkim Manipal Institute of Technology \\ Majitar
}

\begin{abstract}
Edge detection is one of the most commonly used operations in image analysis, which is recently receiving much attention. There are various existing traditional edge detectors which are used to estimate edge magnitude and orientation. The concept of Cellular Automata (CA) also has been used for edge detection making the technique more efficient and effective. This paper includes a comparative study of the traditional techniques with the Cellular Automata (CA) technique which is based on rules of Conway's Game of Life $(\mathrm{GoL})$. The comparative analysis is carried out based on a particular set of assumed parameters which are suitable enough to conclude the best performing edge detector
\end{abstract}

\section{General Terms}

Edge Detection, Cellular Automata, Game of Life, Traditional Edge Detection Methods.

\section{Keywords}

Cellular Automata, Game of Life, Mask Operator, Sobel Edge Detector, Prewitt Edge Detector, Roberts Edge Detector.

\section{INTRODUCTION}

The concept of Cellular Automata (CA) was initiated in the early 1950's by J. Von Neumann and Stan Ulam [3].Afterwards, the CA theory was developed by Stephen Wolfram [3]. Cellular Automata helps in parallel computation, which makes it simpler in comparison to other techniques used in image processing[5][12]. CA can be used for a variety of dynamic systems in different applications. It is a computer algorithm which is discrete in time and space and operates on lattice of sites, here pixels [1][8]. A Cellular Automaton is a model with a collection of cells and has the following characteristics:

Grid -A grid is a collection of two or more infinite sets of evenly-spaced parallel lines at particular angles to each other in a plane. [13][8]

States - Each cell has a state. The number of possible states in $\mathrm{CA}$ is generally finite. The simplest of all the possible states are 1 ("on" or "alive") and 0 ("off" or “dead"). [13]
Neighborhood - The neighborhood of cell, called the core cell(or the central cell),consists of the core cell and those surrounding cells whose states determine the next state of the core cell. The different neighborhood structures for cellular automata are Von Neumann neighborhood (figure 1(a)), Moore neighborhood (figure 1(b)) and Extended Moore neighborhood (figure 1(c)). [13]

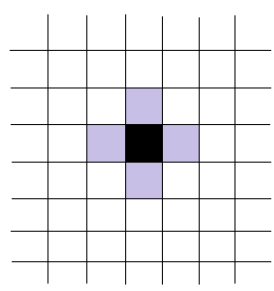

(a)

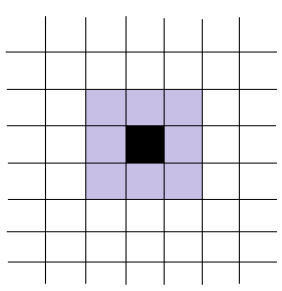

(b)

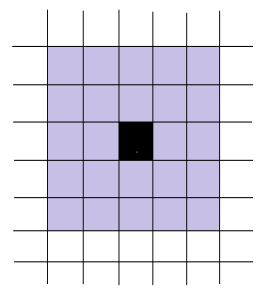

(c)

Figure 1. Neighborhood: (a) Von Neumann Neighborhood (b) Moore neighborhood (c) Extended Moore neighborhood

An edge is a boundary between an object and the background in an image, and it also indicates the boundary between overlapping objects in an image. [2]

Edges of an image are considered a type of crucial information that can be extracted by applying detectors with different methodology [2][9]. Edge detection is one of the most commonly used operations in image processing, computer vision, image understanding system and 
recognizing patterns. It has an important role in image understanding systems [9] [7]. Majority of the classical mathematical methods for edge detection based on the derivative of the pixels of the original image are Gradient operators, Laplacian and Laplacian of Gaussian operators [10]. Gradient based edge detection methods, such as Roberts, Sobel and Prewitt, have used two 2-D or 3-D linear filters to process vertical edges and horizontal edges separately to approximate first-order derivative of pixel values of the image [9][10]. Edge detection also plays an important role in medical field, for example in case of bone fractures, tumour detection, lung cancer diagnosis etc. Other applications of edge detection include analysis of antibiotic images, detection of fabric defects, detection of grain boundaries in rocks, etc [10]. The results of edge detection have many beneficial applications in fields such as image enhancement, recognition, morphing etc [11][6]. The possible advantages of edge detection using cellular automata can be:

Efficiency - Because of its parallel nature, Cellular Automata becomes quite simple and thus is efficient while implementing on hardware.

Global properties - CA is used to work with the grid along with all the cells exhibiting global behaviour and as such can be advantageous over traditional methods which usually provide fragmented output as a result of inconsistent data in the local neighborhood.

Application specificity - For specific applications, rules of cellular automata can be selected accordingly to operate better than general purpose edge detectors.

\section{DIFFERENT EDGE DETECTION TECHNIQUES}

Initially, Sobel and Robert worked with the edge detection. Their work was based on simple intensity gradient operators [11]. On the basis of their work many researchers worked with the development of detectors with good detection performance as well as good localisation performance [11][4]. The three most frequently used edge detection methods that have been used for comparison are Sobel edge detection, Prewitt edge detection and Roberts edge detection.

\subsection{The Sobel Edge Detection}

The Sobel operator performs the two-dimensional spatial gradient measurement on an image and highlights regions of high spatial frequency that corresponds to edges [11][7]. The following figure shows the $3 \times 3$ convolution kernels of the Sobel operator.

\begin{tabular}{|c|c|c|}
\hline-1 & 0 & +1 \\
\hline-2 & 0 & +2 \\
\hline-1 & 0 & +1 \\
\hline
\end{tabular}

\begin{tabular}{|c|c|c|}
\hline+1 & +2 & +1 \\
\hline 0 & 0 & 0 \\
\hline-1 & -2 & -1 \\
\hline
\end{tabular}

The horizontal and vertical edges are detected separately by the Sobel edge detector mask and the directional edges thus produced are combined finally. $G_{x}$ and $G_{y}$ are implemented using convolution masks [11][4]. Taking the convolution results of two mask the magnitude and direction of edge is calculated and given by the following :

$$
\begin{aligned}
& \mathbf{G}=\sqrt{\left(G_{x}{ }^{2}+G_{y}^{2}\right)} \\
& \operatorname{Arg}(G)=\tan ^{-1}\left(\left|G_{y}\right| /\left|G_{x}\right|\right)
\end{aligned}
$$

Sobel operator focuses on pixels that are closer to the centre of the mask. This method detects diagonal edges a well. [11].

\subsection{Roberts Edge Detection}

The Roberts operator performs the two-dimensional spatial gradient measurement on an image and highlights regions of high spatial frequency that corresponds to edges [11]. The following figure shows the $3 \times 3$ convolution kernels of the Roberts operator.

\begin{tabular}{|c|c|c|}
\hline 0 & 0 & 0 \\
\hline 0 & -1 & 0 \\
\hline 0 & 0 & +1 \\
\hline
\end{tabular}

$\mathbf{G}_{\mathbf{x}}$

\begin{tabular}{|c|c|c|}
\hline 0 & 0 & 0 \\
\hline 0 & 0 & -1 \\
\hline 0 & +1 & 0 \\
\hline
\end{tabular}

$\mathbf{G}_{\mathbf{y}}$
Figure 3. Robert operator mask

Diagonal edge gradients are obtained by forming difference of diagonal pairs of pixels. The magnitude form of Roberts cross difference operator is given as follows:

$$
\mathbf{G}_{(\mathrm{x}, \mathrm{y})}=\left|\mathbf{G} \mathbf{1}_{(\mathrm{x}, \mathrm{y})}+\mathbf{G} \mathbf{2}_{(\mathrm{x}, \mathrm{y})}\right|
$$

Whereas in square root means form it can be defined as :

$\mathbf{G}_{(\mathrm{x}, \mathrm{y})}=\left\{\left|\mathbf{G} \mathbf{1}_{(\mathrm{x}, \mathrm{y})}\right|^{2}+\left|\mathbf{G 2 _ { ( x , y ) }}\right|^{2}\right\}^{2}$

Where

$$
G 1_{(x, y)}=F_{(x+1, y+1)}-F_{(x, y)}
$$

and

$$
G 2_{(x, y)}=F_{(x, y+1)}-F_{(x+1, y)}
$$

\subsection{Prewitt Edge Detection}

The Prewitt Edge Detector is used to approximately find out the magnitude and orientation of an edge. The gradient based edge detector is evaluated in the $3 \times 3$ neighbourhood pixels edge gradient operator which is described by the convolution masks as shown [11]:
$\mathbf{G}_{\mathbf{x}}$

\section{$\mathbf{G}_{\mathbf{y}}$}

Figure 2. Sobel operator mask 


\begin{tabular}{|c|c|c|}
\hline+1 & +1 & +1 \\
\hline 0 & 0 & 0 \\
\hline-1 & -1 & -1 \\
\hline
\end{tabular}

$\mathbf{G}_{\mathbf{x}}$

\begin{tabular}{|c|c|c|}
\hline-1 & 0 & +1 \\
\hline-1 & 0 & +1 \\
\hline-1 & 0 & +1 \\
\hline
\end{tabular}

$\mathbf{G}_{\mathbf{y}}$
Figure 4. Prewitt operator mask

The square root edge gradient of Prewitt operator is given by

$$
\mathbf{G}_{(\mathrm{x}, \mathrm{y})}=\left\{\left|\mathbf{G R}_{(\mathrm{x}, \mathrm{y})}\right|^{2}+\left|\mathbf{G C}_{(\mathrm{x}, \mathrm{y})}\right|^{2}\right\}^{2}
$$

With

$G R_{(x, y)=} 1 /(K+2)\left[\left(A_{2}+K A_{3}+A_{4}\right)-A_{0}+K A_{7}+A_{6}\right]$

And

$\mathrm{GC}_{(\mathrm{x}, \mathrm{y})=}=1 /(K+2)\left[\left(\mathrm{A}_{2}+K \mathrm{~A}_{1}+\mathrm{A}_{2}\right)-\mathrm{A}_{0}+K \mathrm{~A}_{5}+\mathrm{A}_{4}\right]$

Where, the value of $\mathrm{K}$ is 1 . To provide unit gain positive weighted and unit loss negative weighted about a separated edge position, the row and column gradients are normalised.

\subsection{Game of Life (GoL)}

Game of life (GoL) was devised by the British mathematician John Horton Conway in 1970 and is a concept of Cellular Automaton. The following transitions occur at each step [8] :

1. Any live cell having less than two live neighbour dies (i.e. under-population).

2. Any live cell having two or three live neighbour lives on to the next step.

3. Any live cell having more than three neighbour dies (i.e. overcrowding).

4. Any dead cell having exactly three live neighbour becomes alive again (i.e. reproduction).

\section{CELLULAR AUTOMATA BASED} ALGORITHM FOR EDGE DETECTION

The algorithm devised for edge detection of images is based on the concepts of cellular automata (states, neighborhood, etc.) and each cell thus changes state based on the rules of Conway's Game of Life:

Variables used - sum : to compute number of neighbors that are alive

$$
\begin{aligned}
& \mathrm{g} 1 \text { : holds intensity of current pixel } \\
& \mathrm{g} \text { : holds intensity of neighboring pixel }
\end{aligned}
$$

Input: Image

Output: Edge detected image with edge pixels marked white Algo:

Begin Read the image into an array of pixel of size [mxn]

\author{
Binarization of image \\ Loop from $\mathrm{i}=1$ to $\mathrm{m}$ \\ Loop from $j=1$ to $n$ \\ $\operatorname{sum}=0$
}

$\mathrm{g}=$ intensity of neighbors

if $g$ is alive then sum $+=1 / /$ to count no. of neighbors alive

Repeat the previous two steps till the four neighbors are read //checking Neumann neighborhood

Check if sum $<=2 / /$ based on GoL rules

if pixel $(i, j)$ is alive then set it to white in the next state

else set it to black

else if sum $=3$ then set pixel $(i, j)$ to white in the next state // based on GoL rules

else set pixel to black in next state

end loop

end loop

end

Here, the image is first read into an array of pixels which makes it suitable for each pixel to be accessed as an element of the array.

The variable sum is incremented each time a neighbor of the pixel is found alive and hence gives the number of neighbors alive. Depending on this number the Conway's Game of Life rules can be applied in order to determine whether this pixel will be alive or dead in the next state.

This processing is done taking each pixel exactly once and thus at each iteration of the entire image we get a new state or next state of the image.

\section{RESULTS AND DISCUSSION}

In this section the results of applying several traditional edge detection methods along with the result produced by the CA based method is shown. The original training data was a set of 20 images of standard size of $256 \times 256$ taken from University of Southern California (USC) SIPI database.

In this comparison study, the parameter taken into consideration for the comparative analysis for any particular image is a set of 10 potential edges that may cause problem in being detected. In the original image the check points (red dots) indicate the edge near to the check point that is to be successfully detected. In the output images, the red check points indicate that the corresponding edge has been successfully detected and the yellow check points indicates edges not detected completely or successfully. 
Image 1 :

The results of image 1 is shown below :

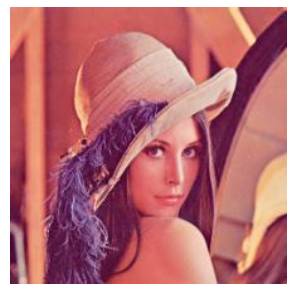

(a)

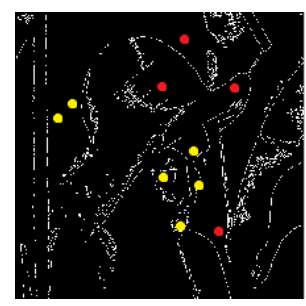

(c)

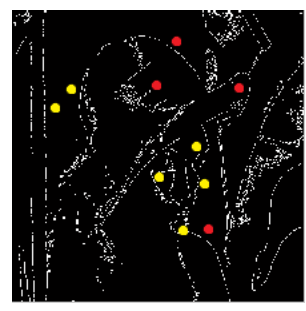

(e)

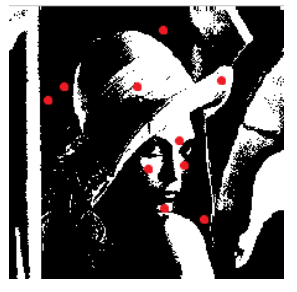

(b)

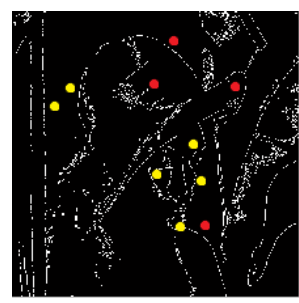

(d)

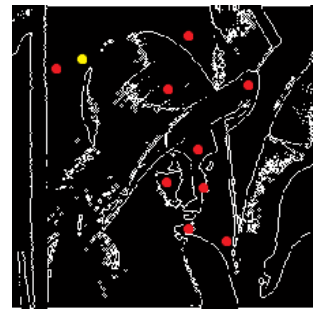

(f)
Figure 5 Results : (a)Original input image, (b)Black and white image, (c)Prewitt Edge Detector, (d)Sobel Edge Detector, (e)Roberts Edge Detector, (f)CA(GoL) based Edge Detector

\section{Image 2 :}

The results of image 2 is shown below :

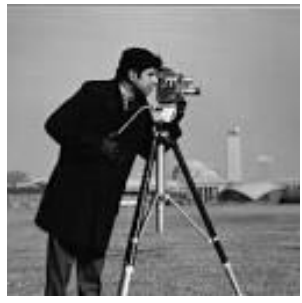

(a)

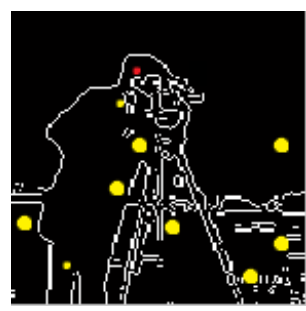

(c)

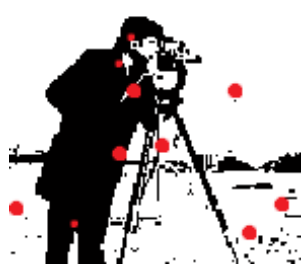

(b)

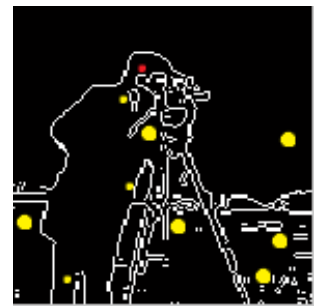

(d)

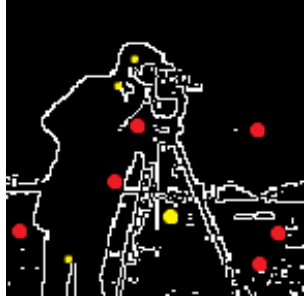

(e)

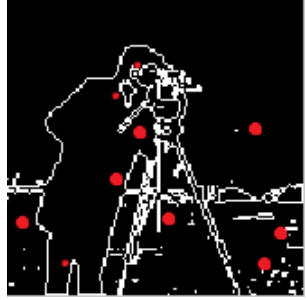

(f)
Figure 6 Results : (a)Original input image, (b)Black and white image, (c)Prewitt Edge Detector, (d)Sobel Edge Detector, (e)Roberts Edge Detector, (f)CA(GoL) based Edge Detector

Image 3 :

The results of image 3 is shown below :

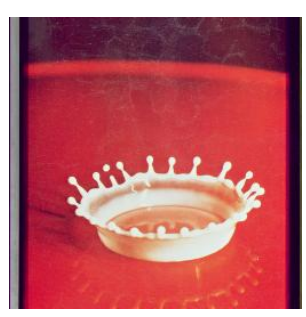

(a)

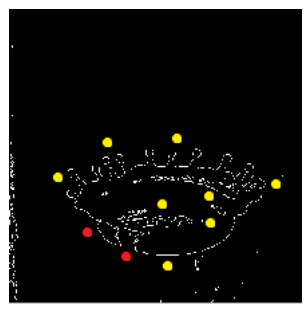

(c)

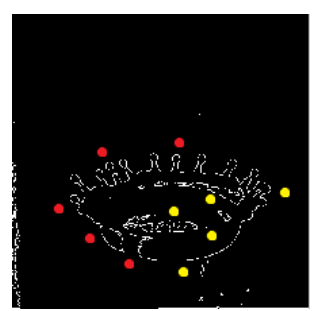

(e)

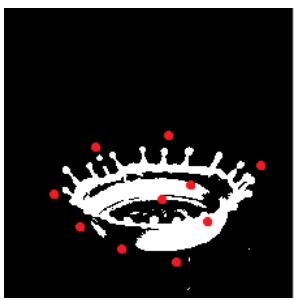

(b)

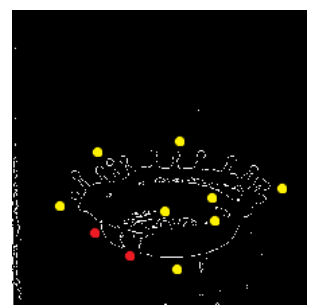

(d)

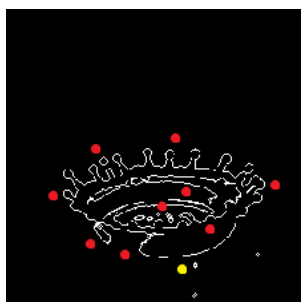

(f)
Figure 7 Results : (a)Original input image, (b)Black and white image, (c)Prewitt Edge Detector, (d)Sobel Edge Detector, (e)Roberts Edge Detector, (f)CA(GoL) based Edge Detector 


\section{Image 4 :}

The results of image 4 is shown below :

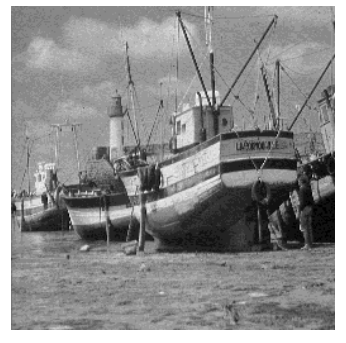

(a)

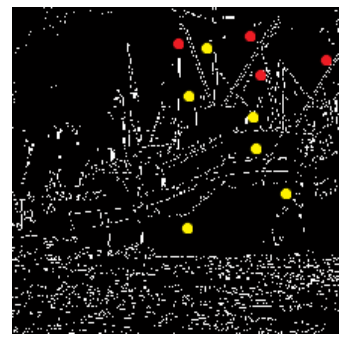

(c)

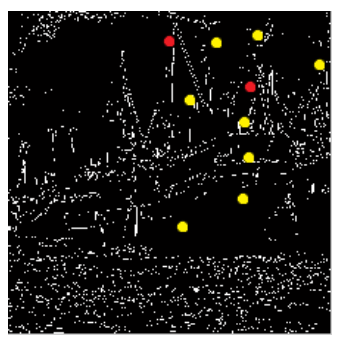

(e)

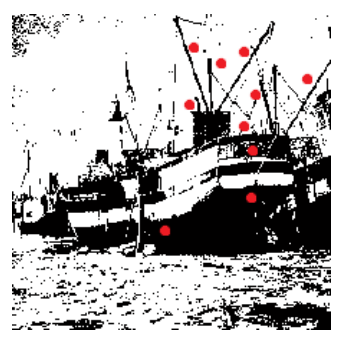

(b)

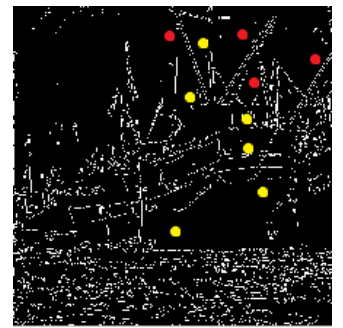

(d)

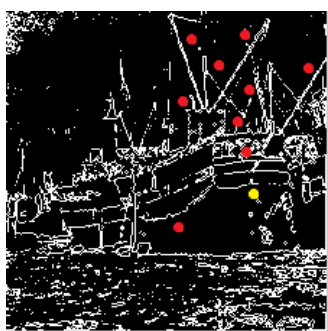

(f)
Figure 8 Results : (a)Original input image, (b)Black and white image, (c)Prewitt Edge Detector, (d)Sobel Edge Detector, (e)Roberts Edge Detector, (f) CA(GoL) based Edge Detector

\section{Image 5 :}

The results of image 4 is shown below :

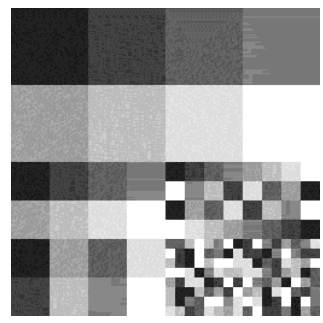

(a)

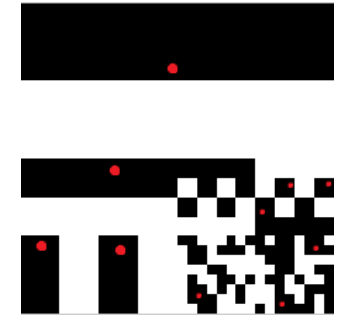

(b)

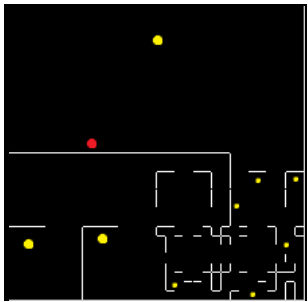

(c)

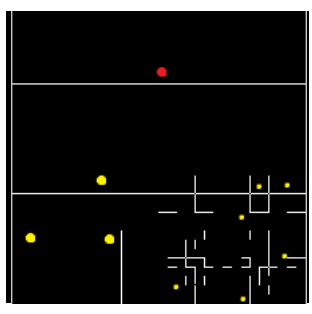

(e)

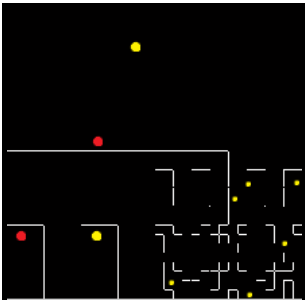

(d)

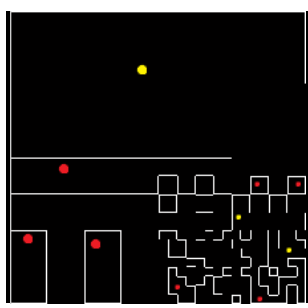

(f)
Figure 9 Results : (a)Original input image, (b)Black and white image, (c)Prewitt Edge Detector, (d)Sobel Edge Detector, (e)Roberts Edge Detector, (f) CA(GoL) based Edge Detector

Image 6:

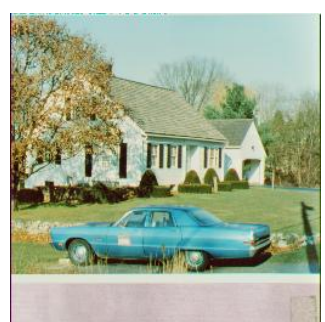

(a)

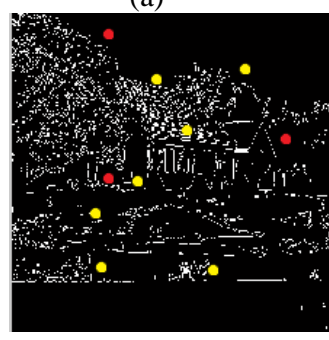

(c)

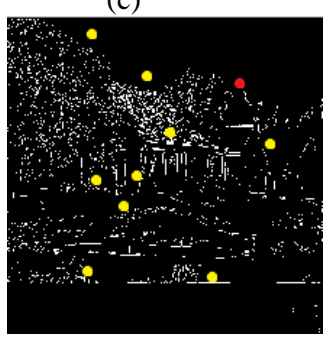

(e)

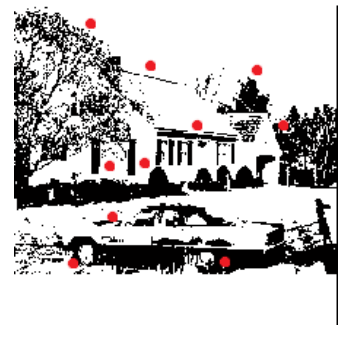

(b)

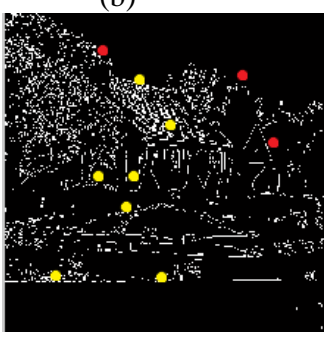

(d)

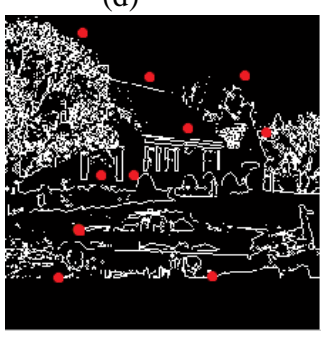

(f)
Figure 10 Results : (a)Original input image, (b)Black and white image, (c)Prewitt Edge Detector, (d)Sobel Edge Detector, (e)Roberts Edge Detector (f)CA(GoL)based Edge Detector 
Table 1. Analysis of outputs produced by traditional methods and CA (GoL) method.

\begin{tabular}{|c|c|c|c|c|c|c|c|c|}
\hline \multirow[b]{2}{*}{$\begin{array}{c}\text { Image } \\
\text { Number }\end{array}$} & \multicolumn{2}{|c|}{ Sobel } & \multicolumn{2}{|c|}{ Prewitt } & \multicolumn{2}{|c|}{ Roberts } & \multicolumn{2}{|c|}{$\begin{array}{l}\text { Cellular Automata } \\
\text { (GoL) }\end{array}$} \\
\hline & $\begin{array}{c}\text { Potential } \\
\text { Edges } \\
\text { successfully } \\
\text { achieved } \\
\text { (out of 10) }\end{array}$ & $\begin{array}{c}\text { Success } \\
\%\end{array}$ & $\begin{array}{c}\text { Potential } \\
\text { Edges } \\
\text { successfully } \\
\text { achieved } \\
\text { (out of 10) }\end{array}$ & $\begin{array}{c}\text { Success } \\
\%\end{array}$ & $\begin{array}{c}\text { Potential } \\
\text { Edges } \\
\text { successfully } \\
\text { achieved } \\
\text { (out of 10) }\end{array}$ & $\begin{array}{c}\text { Success } \\
\%\end{array}$ & $\begin{array}{c}\text { Potential } \\
\text { Edges } \\
\text { successfully } \\
\text { achieved } \\
\text { (out of 10) }\end{array}$ & $\begin{array}{c}\text { Success } \\
\%\end{array}$ \\
\hline Img 1 & 5 & 50 & 6 & 60 & 5 & 50 & 9 & 90 \\
\hline Img2 & 5 & 50 & 6 & 60 & 2 & 20 & 9 & 90 \\
\hline Img3 & 5 & 50 & 5 & 50 & 7 & 70 & 7 & 70 \\
\hline Img4 & 2 & 20 & 2 & 20 & 5 & 50 & 9 & 90 \\
\hline Img5 & 4 & 40 & 4 & 40 & 4 & 40 & 9 & 90 \\
\hline Img6 & 4 & 40 & 4 & 40 & 6 & 60 & 8 & 80 \\
\hline Img7 & 6 & 60 & 6 & 60 & 7 & 70 & 9 & 90 \\
\hline Img8 & 3 & 30 & 4 & 40 & 4 & 40 & 9 & 90 \\
\hline Img9 & 2 & 20 & 2 & 20 & 4 & 40 & 7 & 70 \\
\hline Img10 & 3 & 30 & 3 & 30 & 1 & 10 & 10 & 100 \\
\hline Img11 & 3 & 30 & 4 & 40 & 6 & 60 & 8 & 80 \\
\hline Img12 & 4 & 40 & 3 & 30 & 6 & 60 & 9 & 90 \\
\hline Img13 & 4 & 40 & 3 & 30 & 2 & 20 & 9 & 90 \\
\hline Img14 & 2 & 20 & 1 & 10 & 5 & 50 & 6 & 60 \\
\hline Img15 & 3 & 30 & 4 & 40 & 5 & 50 & 8 & 80 \\
\hline Img16 & 2 & 20 & 1 & 10 & 1 & 10 & 7 & 70 \\
\hline Img17 & 5 & 50 & 4 & 40 & 8 & 80 & 8 & 80 \\
\hline Img18 & 1 & 10 & 1 & 10 & 6 & 60 & 10 & 100 \\
\hline Img19 & 5 & 50 & 5 & 50 & 3 & 30 & 5 & 50 \\
\hline Img20 & 7 & 70 & 7 & 70 & 6 & 60 & 9 & 90 \\
\hline
\end{tabular}

Table 2. Comparison between the Various Edge Detection Methods.

\begin{tabular}{|c|c|c|c|c|}
\hline & \multicolumn{1}{|c|}{ Sobel } & \multicolumn{1}{c|}{ Prewitt } & Roberts & CA(GoL) Method \\
\hline Avg. Success \% & 37 & 37 & 43.5 & 77.5 \\
\hline
\end{tabular}




\section{CONCLUSION}

The experimental results show that on an average Cellular Automata (GoL) based method is approximately $78 \%$ successful in achieving the desired edges i.e., providing sufficiently finer edges even at points or locations where edges may not be detected properly. which is approximately $34 \%$ greater than that of the traditional methods. As a result, it can be concluded that this method provides better results for edge detection in terms of producing finer edges. Also, the output of this method shows very few double edges or broken edges which is an advantage over traditional edge detection methods where problem of double edge and broken edge is observed commonly. Among the traditional methods of edge detection, Robert's detector has been observed to have provided better edge detection than Sobel and Prewitt detectors.

\section{ACKNOWLEDGEMENT}

We would like to express our heartfelt gratitude to, Prof. (Dr.) Kalpana Sharma (HOD, CSE, SMIT), Mr. Biswaraj Sen and Ms. Saswati Bhattacharya (Major Project Coordinators, CSE).

\section{REFERENCES}

[1] Adriana Popovici and Dan Popovici, 2002."Cellular Automata in Image Processing". Proceedings of the $15^{\text {th }}$ International Symposium on the Mathematical Theory of Networks and Systems.

[2] Chang, C., Zhang, Y., Gdong, Y,2004. "Cellular Automata for edge detection of images." Int. Conf. on machine learning and cybernetics 6, pp. 3830-3834.

[3] Deepak Ranjan Nayak and Sumit Kumar Sahu and Jahangir Mohammed, 2013. "A Cellular Automata based Optimal Edge Detection Technique using Twenty-five neighborhood model". , IJCA (0975-8887) volume 84no 10. pp. 27-33.
[4] D. Ziou and S. Tabbone, 1998. "Edge Detection technique and Overview". Pattern Recognition and Image Analysis 8(4). Pp. 537-559.

[5] Fasel Qadir, Khan K.A, 2013. "Investigations of Cellular Automata Linear Rules for Edge detection.". International J. Computer Network and Information Security 3.pp. 47-53.

[6] J. F. Canny. 1986. "A Computational approach to Edge detection”. IEEE Tran. on Pattern analysis and Machine Intelligence PAMI-8.pp. 679-698.

[7] J. V. Neumann. 1966. "Theory of Self-Reproducing Automata". University of Illinois Press.

[8] Nie, Harald, 2006. "Introduction to Cellular Automata : Organic Computing".

[9] Parwinder Kaur Dhillon, IJCA special issue on confluence 2012, the next generation information technology summit. "A Novel Framework to Image Edge Detection using Cellular Automata".

[10] Paul L. Rosin and Xianfang Sun, Springer International Publishing Switzerland 2014. "Chapter 5-Edge Detection using Cellular Automata", pp. 85-103.

[11] Tapas Kumar and G. Sahoo, 2010. "Novel Method of Edge Detection using Cellular Automata.”, International Journal of Computer Applications 9(4), pp. 38-44.

[12] Yang , C., Ye, H., Wang, G, 2002. "Cellular Automata Modelling in Edge Recognition." In: $7^{\text {th }}$ Int. Symp. on Artificial Life and Robotics, pp. 128-132.

[13] Ziou, D. and Tabbone, S., 1998. "Edge Detection Techniques - an overview, pattern recognition and image analysis 8 (4), pp. 537-559. 